\title{
Big Data: Bürgerschreck und Hoffnungsträger! Zusammenfassung und Fazit
}

Jeder von uns hinterlässt persönliche Daten, wenn er online ist. Ganz gleich, ob sparsam oder ungehemmt, gewollt oder ungewollt, anonym oder offenherzig - alles bleibt. Fotos oder Text, Emojis oder Likes - alles dient zum Re-Identifizieren. Massenhaft entstehen Datenmengen, deren Ausmaß kaum vorstellbar ist und die mit dem Begriff ,Big Data' ebenso einfach wie euphemistisch beschrieben werden. Noch entstehen diese Daten vor allem dann, wenn ein onlinefähiges Endgerät eingeschaltet wird. Aber selbst das wird nicht mehr benötigt. Daten entstehen im wahrsten Sinne des Wortes auf Schritt und Tritt, in den eigenen vier Wänden genauso wie beim Fahren auf der Autobahn, ohne dass irgendjemand dafür ein Smartphone einschalten muss. Man muss kein Prophet sein, um vorherzusagen, dass Massendaten weiter exponentiell anwachsen werden. Die nächste Dekade wird zweifelsohne die Dekade von Big Data, in der wir beantworten müssen, wie wir damit umgehen und was wir damit machen.

Big Data ist dabei weder die Sache einiger weniger Unternehmen noch ein Thema für ausgewählte IT-Experten. Das ergibt sich aus der Natur der Sache, nämlich der Tatsache, dass Big Data aus den Online-Beziehungen bzw. ganz generell aus dem Handeln und Verhalten der Nutzer entstehen, mithin jeden einzelnen unmittelbar involvieren, so oder so. Wenn es also darum geht zu entscheiden, wie mit diesen Massendaten umzugehen ist, wer sie erhebt, speichert, handelt und für welche privaten, sozialen und öffentlichen Zwecke sie von wem eingesetzt werden, dann ist das ein Themenfeld, das der öffentlichen und politischen Meinungsbildung genauso unterliegt wie den ökonomischen Gesetzen des Marktes.

Wie bei allen Dingen, die nur schwer vorstellbar sind und sich den Alltagswahrnehmungen entziehen, ist jedoch genau diese Meinungsbildung mit erheblichen Schwierigkeiten behaftet. Wer kann schon sagen, wie man sich Big Data 
ganz konkret vorzustellen hat? Und wenn man es könnte, würde es bei der Beurteilung weiterhelfen? Wohl eher nicht. Ohne ein kommunikativ verwertbares Bild vor Augen lässt sich aber schwer diskutieren und debattieren. Kein Wunder also, dass die öffentliche Meinungsbildung zu Big Data zwischen dystopischen Schreckensvisionen einerseits und utopischen Heilserwartungen andererseits changiert, d. h. unvorhersehbar schwankt, wobei die Schreckgeschichten dominieren. Deshalb ist es auch kein Wunder, wenn Politik und Gesetzgeber sich bislang darauf konzentriert haben, den Bürger vor dem Missbrauch seiner persönlichen Daten zu schützen.

Wer aber einen weitergehenden gesellschaftlichen Diskurs führen will, in dem nicht nur die Risiken von Big Data, sondern auch die Chancen ausgewogen betrachtet werden, der muss dickere Bretter bohren. Denn schon die kleinste Veränderung im vorherrschenden Datenschutz-Paradigma erweist sich als höchst schwierig und ist nur in kleinen Schritten denkbar. Vor allem der Blick auf alltagsnahe Lebenswelten, in denen erkennbar wird, was Big Data und selbstlernende Algorithmen beispielsweise für den Fortschritt in Sachen Gesundheit, Wohnen oder Mobilität konkret bringen, kann helfen, Big Data aus dem Abseits der Abstraktion zu holen und damit eine breitere Diskussionsgrundlage zu schaffen. Doch was kommt davon wirklich beim umsorgten und geschützten Bürger an, der ja immer zugleich auch Onlinekunde, App-Junkie oder Social-Media-Nutzer ist? Und wie können oder müssen sich Unternehmen $\mathrm{zu}$ alledem verhalten, deren Geschäft auf Big Data und deren Auswertung durch Algorithmen beruht?

\subsection{Zur Gestaltung des öffentlichen Diskurses über Chancen und Risiken von Big Data: Die Ergebnisse im Überblick}

Die hier vorgelegte wissenschaftliche Studie hat sich mit diesen Themen- und Fragestellungen befasst, und zwar interdisziplinär und mit unterschiedlichen qualitativen und quantitativen Methoden, so wie es das Thema Big Data mit seinen vielfältigen Facetten verlangt. Kap. 1 betrachtet den öffentlichen Diskurs über Big Data und gibt einen Überblick über politische Handlungsoptionen. Darauf aufbauend zeigt Kap. 2 konkrete Anwendungen von Big Data und Künstlicher Intelligenz in relevanten Lebenswelten und analysiert die Auswirkungen auf bestehende Geschäftsmodelle am Beispiel von Versicherern. Schließlich wird in Kap. 3 auf der Grundlage einer repräsentativen Erhebung der Frage nachgegangen, was von alledem bei den Bürgern ankommt und welche Schlussfolgerungen daraus zu ziehen sind. 
Dies sind die wichtigsten Ergebnisse im Überblick:

- Der öffentliche, in den Medien ausgetragene, Diskurs zum Umgang mit Massendaten (Big Data) ist von Erzählungen dominiert, die um Konflikte und Kollisionen kreisen und nicht über diese hinauskommen. In der Medienanalyse sind sie als Varianten des Big Brother-Narratives einzuordnen und haben ganz überwiegend den Charakter von Dystopien.

- Diese Dominanz der negativen Narrative ist auch in der Bevölkerungsumfrage deutlich zu erkennen. Es überwiegt eine skeptische bis ablehnende Grundeinstellung gegenüber Big Data. Risiken sind in den Köpfen der Befragten gegenüber Chancen überrepräsentiert. Die Dominanz des Big Brother-Narratives behindert dementsprechend eine breit getragene Verständigung darüber, wie sich die Risiken von Big Data beherrschen lassen, ohne die Chancen unangemessen zu reduzieren.

- Der politische Diskurs ist ganz im Gegensatz zum Mediendiskurs weiterhin von optimistischen, ja idealistischen Grundannahmen geprägt. Tradierte Idealtypen wie das Aufklärungsideal des mündigen Bürgers oder das Menschenbild des Homo oeconomicus, des rationalen Marktteilnehmers, sind nach wie vor die basalen Leitbilder von Politik bzw. Gesetzgebung, denen mit geeigneten Maßnahmen, nicht zuletzt einer detaillierten Rechtsetzung, Geltung zu verschaffen ist.

- Das Bild des weitgehend rational denkenden und agierenden Bürgers lässt sich jedoch schon lange nicht mehr aufrechterhalten und wird auch in unserer Studie widerlegt. Bedingungen eines weitgehend rationalen, selbstbestimmten Handelns sind Wissen, Können sowie ausreichend Motivation, beides auch einzusetzen. Zwar sind Grundbegriffe der Digitalisierung und der Künstlichen Intelligenz in der Bevölkerung angekommen, und ein Großteil fühlt sich zumindest in Teilen kompetent. Aber Wissensdefizite bestehen gerade in den Themen, die die Vernetzung von Daten behandeln und damit den souveränen Umgang mit den persönlichen Daten besonders tangieren, z. B. Big Data, Telematik, Internet of Things und e-Privacy. Die Bürger fühlen sich quer durch alle Bevölkerungsgruppen - aufgrund von Komplexität und Marktstrukturen nur sehr bedingt handlungsfrei oder souverän.

- Augenfällig ist, dass die Mehrheit der Nutzer kaum auf die Furchtappelle der Big-Brother-Narrationen durch entsprechende Verhaltensänderungen reagiert. Sie blicken einerseits skeptisch auf Big Data, konsumieren ausgiebig dystopische Big-Brother-Geschichten und messen dem Datenschutz nach wie vor hohe Relevanz zu. Andererseits gehen sie sorglos oder fatalistisch mit ihren Daten um und nehmen angebotene Informations- oder Schutzmöglichkeiten 
nicht oder nur begrenzt wahr. Besorgte Bürger, aber sorglose Verbraucher das Nutzer-Paradoxon findet auch in unserer Befragung Bestätigung. Datenschutzbedenken und Sorge vor Big Data halten kaum davon ab, als nützlich oder notwendig empfundene Dienste in Anspruch zu nehmen, und zwar ziemlich unabhängig vom digitalen Wissen und von der Einstellung zum Datenschutz.

- Die Bevölkerung ist im Netz und das in doppeltem Sinne: Das Internet wird intensiv und über unterschiedlichste Geräte genutzt. Zugleich sind die Bürger über zahlreiche Dienste und Geräte ,im Netz ' der Dienste und hinterlassen so umfangreiche Datenspuren. Dabei dominieren die US-amerikanischen Internetgiganten, die zumindest bis zur Inkraftsetzung der EU-Datenschutzgrundverordnung im Mai 2018 deutlich niedrigeren Datenschutzstandards unterlagen als ihre deutschen Wettbewerber. Reichweite und Nutzenumfang der globalen Player dominieren offenbar gegenüber Nähe und Datenschutz. Die Bürger fühlen sich in hohem Maße ausgeliefert und nutzen trotzdem munter die ,Frightful 5'.

- Insgesamt bleibt es bei der Dichotomie der Rollen und Einstellungen, die zu dem besagten Nutzer-Paradoxon führen: Der Nutzer als Bürger ist skeptisch, schutzbedürftig und kulturpessimistisch, als Verbraucher ist er sorglos, bequem und pragmatisch. Man könnte diese Beobachtung auch positiver formulieren: Das tatsächliche Verhalten der Bürger im Netz orientiert sich deutlich stärker an den Chancen von Big Data als an den Risiken.

- Dabei ,hilft' digitale Bildung, denn sie führt laut Gruppenvergleichen in unserer Befragung dazu, dass die meisten Sorgen kleiner werden und die Chancen insgesamt stärker in den Fokus rücken. Wenn dann noch anstelle abstrakter Risiken und Chancen über ganz konkrete, nutzenstiftende Anwendungsfelder gesprochen wird, dann wandelt sich das Bild, und Big Data stößt in vielen Einsatzbereichen auf hohe Akzeptanz. Das erstreckt sich auf Smart Services aller Art bis hin zu Sicherheitsfragen, Kriminalitätsbekämpfung und Verkehrsüberwachung.

- Es herrscht breiter Konsens bezüglich Fairness und Nachvollziehbarkeit als Grundanforderung an datengestützte Systeme. Dies impliziert Transparenz, Vertrauen in (oder externe Kontrolle der) Anbieter, aber auch Neutralität, Diskriminierungsfreiheit, Datenqualität und natürlich Datensicherheit. All das sollen die agierenden Unternehmen und die Aufsichtsinstanzen gleichermaßen gewährleisten. Dann, so diese normative Sichtweise, kann auch die Akzeptanz von Big Data als Hoffnungsträger steigen. Doch ganz so einfach ist es nicht. 
Vor allem bei unstrukturierten Daten (z. B. Fotos, Videos, Sprache), selbstlernenden Algorithmen oder Blockchains ist es schwer bis nahezu unmöglich, die normativen Vorgaben der diskriminierungsfreien Datenqualität und allgemeiner Nachvollziehbarkeit ganz praktisch zu erfüllen.

- Die Bürger wünschen sich vielleicht deshalb weiterhin eine klare Reglementierung und Regulierung durch den Staat. Dennoch ist unübersehbar, dass die Rechtsetzung im Kontext einer dynamischen Digitalisierung bereits an ihre Grenzen gestoßen ist. Selbstlernenden Algorithmen mit immer diffizileren juristischen Befehlsfolgen zum Datenschutz begegnen zu wollen, ist schon für sich keine wirklich einlösbare Schlussfolgerung. Darüber hinaus führt das eben beschriebene paradoxe Nutzerverhalten dazu, dass Regelungen z. B. aus der EU-DSGVO, die eigentlich Wahlfreiheiten und somit Konsumentensouveränität sicherstellen sollen, ins Leere zu laufen drohen.

- Umgekehrt kann das volle Potenzial datengetriebener Services erst dann entfaltet werden, wenn möglichst viele Daten unterschiedlicher Quellen generiert, auch ohne einengende Zweck- und Planvorgaben ausgewertet und miteinander kombiniert werden. Die Wirkmächtigkeit der Korrelationen wird sich in der Big- Data-Dekade erst noch voll entfalten. Datensparsamkeit, Zweckbindung von Daten und Datenlöschungen, wie sie die EU-DSGVO aus Datenschutzerwägungen verständlicherweise fordern, sind vor diesem Hintergrund kontraproduktiv. Diese gesetzlichen Regelungen stehen infolgedessen zugleich im Widerspruch zur Entfaltung künstlicher Intelligenz.

- Mit diesen Restriktionen lassen sich auch die positiven Beiträge von Big Data und Künstlicher Intelligenz für gesellschaftlich relevante Zwecke nur unzureichend realisieren. Im Mittelpunkt stehen dabei Zwecke des Gemeinwohls (in der angelsächsischen Diskussion heißt dies , social goods"). Big Data sowohl aus öffentlicher als auch privater Hand bieten ungeahnte Chancen, die gravierendsten Probleme der Welt nachhaltig, d. h. vor allem auf globaler Reichweite, zu lösen. Das beginnt bei Klimaschutz, geht über Gesundheit und Ernährung bis hin zu Bildung. Akzeptierte Regeln zu finden, mit denen Zugang und Einsatz von Big Data für Gemeinwohlzwecke erreicht werden kann, steht deshalb international auf der politischen Agenda.

- Die Open-Data-Bewegung ist dabei, dieses Terrain zu erobern. Die Idee einer Daten-Allmende (,Commons'), in der öffentliche Massendaten nach definierten Regeln der Nachhaltigkeit zwischen Organisationen aller Art geteilt werden, ist ebenso faszinierend wie ganz praktisch umzusetzen, wie dies beispielsweise offene Datenbestände aus öffentlichen Verwaltungen oder 
Forschungseinrichtungen aller Art zeigen. Noch weitergehender ist der Gedanke einer nach ethisch verantwortbaren Zwecken differenzierten DatenInfrastruktur, für deren Sicherheit und Qualität dann sogar ein öffentlicher Auftrag besteht. Bemerkenswerterweise finden solche Ideen in unserer Umfrage eine beachtliche Zustimmung.

- Nicht zuletzt aufgrund der Grenzen des Datenschutzkonzeptes ist es sinnvoll, eine Verständigung über den ausgewogenen Umgang mit Big Data auf einem anderen Spielfeld bzw. mit einer anderen Agenda zu suchen. Das Konzept der Datenethik ist - insbesondere in Verbindung mit dem Open Data-Prinzip so ein alternatives Spielfeld. Hier geht es darum, den Bürger in seiner Rolle als Nutzer digitaler Technologien dabei zu unterstützen, dass er seine Daten gezielt zu den von ihm gewünschten Zwecken weitergeben kann. Der Nutzer ist weniger Schutzobjekt, sondern als Datengeber, Datenspender oder gar Datenhändler Subjekt der Digitalisierung. Datenethik ersetzt das Datenschutzparadigma nicht, sondern baut auf den erreichten Schutzstandards auf und erweitert sie um ein echtes Handlungskonzept. Es weist nicht nur den Nutzern eine andere, nämlich aktive und gestaltende Rolle zu, sondern allen Akteuren rund um Big Data. Im Mittelpunkt stehen dabei die Chancen, die sich aus Big Data ergeben, die der Nutzer mit seinen Daten selbst aktiv unterstützen kann.

- Schon jetzt lässt sich sagen, dass Unternehmen, deren Umgang mit von ihnen gesammelten und genutzten Daten auf klaren ethischen Handlungsgrundsätzen beruht, die Gewinner dieser Entwicklung sein werden. Unternehmen sind nicht mehr nur zur Compliance mit den Datenschutzgesetzen aufgefordert. Sie werden darüber hinaus ethisch verpflichtet, die Chancen von Big Data bzw. Künstlicher Intelligenz proaktiv zu nutzen, und zwar sowohl für privatwirtschaftliche als auch soziale Zwecke. Corporate Digital Responsibility lässt sich schon jetzt als neue Dimension einer verantwortungsvollen Unternehmensführung ausmachen.

- Ordnungspolitisch motivierte Konzepte, die nicht zuletzt auf die Geschichte der ,Frightful 5' Bezug nehmen, können die Vorstellung des souverän mit seinen Daten agierenden Nutzers wirkungsvoll flankieren. Das gilt beispielsweise für einen unternehmens- und branchenübergreifenden Datenaustausch (Daten-Sharing), zu dem Daten-Oligopole ggf. gezwungen werden können. Wettbewerb der Algorithmen statt Oligopol der Datenkraken - diese Geschichte scheint auch für die Bevölkerung interessant zu sein. Kartellrechtliche Maßnahmen werden ausdrücklich begrüßt, aber auch der Ansatz eines verbindlichen Daten-Sharings könnte zukünftig überzeugen. 


\subsection{Zum Nutzen von Big Data in konkreten Lebenswelten: Die Ergebnisse im Überblick}

Big Data kann substanzielle Mehrwert für die Bürger in ihren Lebenswelten generieren. In Bezug auf die Lebenswelten Wohnen, Gesundheit und Mobilität wurden Einsatzbereiche von Big Data dargestellt und beispielhaft an der Versicherungswirtschaft analysiert, wie weit sich daraus die Rolle einer Branche in der Gesellschaft ändern kann:

- Auf Basis von Big Data werden intelligente, digitalisierte Angebote ermöglicht, mit denen Bedürfnisse der Bürger erfüllbar sind (Smart Services). Smart Services erknüpfen Produkte, Dienstleistungen und Prozesse, die dem Kunden als individualisiertes Gesamtangebot und , as a service' zur Verfügung stehen. Im Mittelpunkt der Smart Services steht der Bürger mit seinen Wünschen und Bedürfnissen, die oftmals nicht durch einzelne Anbieter und deren Produkte erfüllt werden können. Zentral für nutzenstiftende Angebote in den Lebenswelten der Bürger ist daher der ganzheitliche Ansatz digitaler Ökosysteme.

- Datengetriebene Angebote in der Lebenswelt Wohnen schaffen ein Smart Home, in dem die Wohnumgebung komfortabler genutzt, eine erhöhte Sicherheit innerhalb des Hauses sowie ein verbesserter Schutz nach außen gewährleistet und der Ressourcenverbrauch effizienter gesteuert werden kann. Künftig ist mit ganzheitlichen Lösungen für das Smart Home des Bürgers zu rechnen. Das hat auch Konsequenzen für Versicherer, da es in größeren Netzwerken (Ökosystemen) künftig reichen könnte, daran jeweils nur einen Versicherer exklusiv zu beteiligen.

- Im Mittelpunkt der Lebenswelt Gesundheit stehen die Sammlung von Daten und deren Einsatz zur Verbesserung von Prävention, Diagnose und Therapie von Krankheiten. Versicherer könnten wegen ihres bereits vorhandenen Wissens über Krankheitsverläufe und das verhältnismäßig hohe Vertrauen, das Bürger ihnen bezüglich des Umgangs mit persönlichen Daten entgegenbringen, zum Orchestrator des Datenmanagements von Ökosystemen in der Lebenswelt Gesundheit werden. Durch die hohe Sensibilität der Daten geht damit eine besonders große Verantwortung einher.

- Drei Trends prägen die Lebenswelt Mobilität: die Sharing Economy, die Entwicklung zum autonomen Fahren und die intermodale Verknüpfung von Verkehrsmitteln. Das hat u. a. zur Folge, dass der Gesamtbestand zu versichernder Kraftfahrzeuge zurückgeht und sich die klassischen Deckungskonzepte nicht 
mehr ohne Weiteres anwenden lassen. Die große Herausforderung der Versicherungswirtschaft besteht angesichts großer (Automobilhersteller) und teils neuer (Big Techs) Wettbewerber mit ihren Smart Services darin, den Kundenzugang zu erhalten und mit den neu gewonnenen Mobilitätsdaten echte Mehrwert für die Kunden und Nutzenpotenziale auch jenseits einer für einzelne Zielgruppen günstigeren Tarifierung und Prämieneinstufung zu erzeugen.

- Die Bürger wertschätzen den Mehrwert, den diese Smart Services ihnen bieten können. Die Grundskepsis gegenüber Big Data kippt, wenn konkrete, nutzenstiftende Anwendungen genannt werden. Ein Großteil unserer Befragten kann sich eine Vielzahl von Anwendungen in den drei Lebenswelten Mobilität, Wohnen und Gesundheit durchaus vorstellen. Analog zu den gesellschaftlichen Einsatzfeldern ist die Akzeptanz besonders dann hoch, wenn es um die Abwendung von Gefahren geht - sei es im Feld der Gesundheit, beim Einbrecherschutz oder selbst zur Überwachung der Verkehrsregeln.

- Anwendungsfelder werden in der Tendenz umso negativer bewertet, je mehr sie den Alltag nicht nur unterstützen, sondern gefühlt die Kontrolle übernehmen. Werden aber keine als wesentlich empfundenen Handlungsspielräume eingeschränkt und ist der Nutzen offensichtlich, ist auch die Akzeptanz weitestgehend automatischer Steuerung hoch.

- Viele der Anwendungen in den genannten Lebenswelten (zum Beispiel im Gesundheitsmonitoring, der Verkehrssteuerung oder auch im ,Smart Home') erfordern eine weitgehende, Überwachung' des Einzelnen. In den Antworten der Befragung lässt sich kaum ein klarer Zusammenhang zwischen dem Grad an Überwachung und der Akzeptanz des jeweiligen Angebotes feststellen. Vielmehr scheint - wiederum in Einklang mit dem Nutzerparadoxon - alleine der empfundene Nutzen über Befürwortung oder Ablehnung zu entscheiden.

- Mit neuen digitalen Lösungen ändert sich das Geschäftsmodell der Versicherer grundlegend. Durch den Einsatz von Sensoren können Schäden vorhergesehen und so zunehmend auch vermieden werden (Reduzierung klassischer Risiken). Auf der anderen Seite scheint es im Hinblick auf die wachsende Bedeutung der Vernetzung und der steigenden Relevanz von Daten- und Prozesssicherheit angezeigt, neu entstehende Kumulrisiken z. B. durch IT-Ausfälle oder Hackerangriffe abzusichern sowie für dabei entstehende Kosten aufzukommen (Entstehung neuer Risiken).

- Überhaupt stellt sich die Frage, welche neuen Rollen den Versicherern im Big Data-Zeitalter zuzuschreiben sind. Wie schon im Zusammenhang mit dem Datenethikkonzept erwähnt, liegt gerade in neuen Rollenbeschreibungen bzw. Zuschreibungen jenseits der eingefahrenen Routinen womöglich der Schlüssel für einen chancenorientierteren Umgang mit Big Data und Künstlicher 
Intelligenz. Für Versicherer können solche neue Rollenmodelle jenseits der tradierten Rolle des Kostenerstatters etwa so aussehen:

- ,Retter' oder ,Schadenmanager': Ist ein Schaden eingetreten, tritt neben den eigentlichen finanziellen Schadenausgleich immer mehr das ,Management ' des Schadens, also z. B. die Organisation von Reparaturen oder medizinischen Therapien. Mit zunehmender Vernetzung ergeben sich hier weitergehende Möglichkeiten, sowohl die Schäden zu erkennen als auch die Maßnahmen zu koordinieren und zu begleiten.

- ,Coach" oder ,Schadenverhüter': Ebenso ermöglichen Vernetzung, die Analyse großer Datenbestände und der Einsatz von Sensoren den Versicherern zunehmend, schon vorab mögliche Risiken zu erkennen und durch Aufklärung oder Verhaltensfeedbacks zu minimieren.

- ,Kontrolleur' oder ,Motivator': Wenn Risiken vorausschauend gemessen und erkannt werden können, dann ist auch eine unmittelbare Einflussnahme auf das Verhalten des Versicherungsnehmers durch positive oder negative Sanktionen nicht mehr weit. Dies kann zum Beispiel über die Bepreisung erfolgen, aber auch in weitergehenden konkreten Handlungsvorschriften oder Verboten, welche wiederum durch Sensoren und Vernetzung überprüft werden können.

- Die Ergebnisse der Umfrage zeigen, dass die Kunden durchaus aufgeschlossen für eine solche Rollenerweiterung ihrer Versicherer sind. Fast alle der exemplarisch genannten Angebote werden von einem bis zu zwei Dritteln der Befragten begrüßt. Umgekehrt lehnen nur zwischen 11 und $37 \%$ der Befragten die entsprechenden Angebote ab. Lediglich für eine laufende Analyse des Fahrstils fällt die Akzeptanz deutlich geringer aus.

- Ob die Menschen eher die Rolle des Versicherers als Schadenmanager, als Schadenverhüter oder als Kontrolleur befürworten, lässt sich nicht eindeutig beantworten. Vielmehr hängt die Akzeptanz von der konkreten Ausgestaltung der Angebote innerhalb der jeweiligen Rolle ab.

- Ebenso wenig besteht ein eindeutiger Unterschied zwischen den Lebenswelten. Die erweiterten Rollen fanden in unserer Befragung sowohl bezogen auf Mobilität als auch auf Gesundheit und Wohnen Akzeptanz, wobei die Zustimmung im besonders datensensiblen Bereich der Gesundheit insgesamt sogar noch etwas höher ausfiel.

- Inwieweit sich daraus eine wirklich breite Nachfrage im Markt entwickelt, hängt von der konkreten Ausgestaltung der Angebote ab, bei denen es besonders wenn auch Sanktionen im Spiel sind - darauf ankommt, dass die zugrunde liegenden Merkmale und Algorithmen nachvollziehbar und gerecht erscheinen. Verhaltensmerkmale, die durch Big Data gemessen werden und 
möglichen Sanktionen durch den Versicherer zugrunde liegen, sollen durch den Kunden tatsächlich zu beeinflussen sein. Schließlich gilt auch in diesem Zusammenhang wieder: Die persönlichen Vorteile müssen offensichtlich und der Datenschutz muss gewährleistet sein.

- Gerade die Anforderungen der Transparenz und der Nachvollziehbarkeit stellen jedoch - wie schon ausgeführt - im Kontext von Big Data und künstlicher Intelligenz schwer erfüllbare Bedingungen dar. Vor allem selbstlernende Algorithmen im Rahmen der Künstlichen Intelligenz sind kaum noch durchschaubar, geschweige denn vermittelbar. Hier ist eine Balance zwischen mathematischer Genauigkeit einerseits und breiter Vermittelbarkeit auf der anderen Seite anzustreben.

Damit schließt sich der Kreis. Sowohl der beschriebene politische und ökonomische Paradigmenwechsel als auch die neuen Rollenzuschreibungen an nahezu alle Akteure werden umso erfolgreicher sein, je besser es gelingt, sie mit wirksamen Narrationen bzw. Narrativen zu verbinden, die nicht in Konflikten und Kollisionen hängen bleiben und die Komplexität des Themenfelds im Sinne einer breiten Verständigung sinnvoll reduzieren. Genau für diese Konstruktion von gesellschaftspolitisch relevanten Erzählungen eignen sich sowohl die genannten Überlegungen zur Datenethik sowie zur Daten-Infrastruktur als auch die Betrachtung der konkreten digitalen Lebenswelten grundsätzlich gut. Anwendungen auf Basis von Big Data haben aus technischer Sicht ein hohes Potenzial, Motive wie Sicherheit, Komfort, Effizienz oder Kontrolle zu bedienen und somit einen hohen direkten Nutzen für die Bürger zu generieren. Positive, mit Assoziationen aufgeladene Codes könnten diese Seiten von Big Data in der Vorstellungswelt der Menschen verankern und so helfen, ein etwas balancierteres Bild der neuen Datenwelt zu schaffen.

Bisher dominiert aber das Big-Brother-Narrativ mit seinen Abwandlungen die öffentliche Big-Data-Arena, neben dem bisher allenfalls die Vorstellung vom ,Forscher und Entwickler' sowie vom praktischen ,Alltagsassistenten " eine zumindest erwähnenswerte Statistenrolle einnehmen konnte. Zur gesellschaftlichen Konstruktion positiver, sinnstiftender Narrationen, die den Schutz des Bürgers vor Datenmissbrauch einerseits und den Nutzen von Big Data andererseits besser ausbalancieren helfen, ist es also zumindest noch ein weiter Weg. 
Open Access Dieses Kapitel wird unter der Creative Commons Namensnennung 4.0 International Lizenz (http://creativecommons.org/licenses/by/4.0/deed.de) veröffentlicht, welche die Nutzung, Vervielfältigung, Bearbeitung, Verbreitung und Wiedergabe in jeglichem Medium und Format erlaubt, sofern Sie den/die ursprünglichen Autor(en) und die Quelle ordnungsgemäß nennen, einen Link zur Creative Commons Lizenz beifügen und angeben, ob Änderungen vorgenommen wurden.

Die in diesem Kapitel enthaltenen Bilder und sonstiges Drittmaterial unterliegen ebenfalls der genannten Creative Commons Lizenz, sofern sich aus der Abbildungslegende nichts anderes ergibt. Sofern das betreffende Material nicht unter der genannten Creative Commons Lizenz steht und die betreffende Handlung nicht nach gesetzlichen Vorschriften erlaubt ist, ist für die oben aufgeführten Weiterverwendungen des Materials die Einwilligung des jeweiligen Rechteinhabers einzuholen.

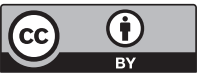

